\title{
Queen mandibular pheromone modulates hemolymph ecdysteroid titers in adult Apis mellifera workers
}

\author{
Ashton M. Trawinski ${ }^{1,2}$, Susan E. FAHRBACH ${ }^{1,2}$ \\ ${ }^{1}$ Department of Biology, Wake Forest University, 1834 Wake Forest Road, Winston-Salem, NC 27109, USA \\ ${ }^{2}$ Center for Molecular Signaling, Wake Forest University, 1834 Wake Forest Road, Winston-Salem, NC 27109, USA
}

Received 14 July 2017 - Revised 24 November 2017 - Accepted 3 January 2018

\begin{abstract}
To test the hypothesis that exposure to queen mandibular pheromone (QMP) modulates ecdysteroid production in adult worker honey bees, ecdysteroids were measured in hemolymph and other tissues of individual adult worker honey bees reared with or without QMP in cages and field colonies. Ecdysteroid titers were higher in caged workers exposed to QMP continuously from the first day of adult life than in workers reared without QMP. Statistical cluster analysis suggested the possibility that a subgroup of workers ("responders") is more sensitive to QMP in this regard than other workers. In 12-day-old workers, ecdysteroid titers in workers reared in queenright $(\mathrm{QR})$ colonies were similar to those observed in cages with QMP, but lower than those in queenless $(\mathrm{QL})$ colonies. Differences in number of ovarioles or degree of ovarian activation did not correlate with hemolymph ecdysteroids. Previous studies have demonstrated ecdysteroids in hemolymph in very young adult workers and in workers in QL colonies; the present study indicates that production of ecdysteroids occurs in older adult worker honey bees in the absence of morphological signs of ovarian activation, with cage studies revealing a modulatory role for QMP masked in the complex environment of the hive.
\end{abstract}

ecdysteroids / ovary / queen mandibular pheromone / queenless / queenright

\section{INTRODUCTION}

Insects produce ecdysteroid hormones (Niwa and Niwa 2014). The source of ecdysteroids in larvae and pupae is the prothoracic gland, which degenerates after metamorphosis (Snodgrass 1956; Dai and Gilbert 1997). The site of steroid synthesis in females then shifts to ovarian follicle cells (Lafont et al. 2005; Brown et al. 2009). The primary steroid produced by insect ovaries is ecdysone $\left(\mathrm{C}_{27} \mathrm{H}_{44} \mathrm{O}_{6}\right)$, which is converted to 20 hydroxyecdysone (20E) by peripheral tissues such

Electronic supplementary material The online version of this article (https://doi.org/10.1007/s13592-018-0562-6) contains supplementary material, which is available to authorized users.

Corresponding author: A. Trawinski, trawas01@gmail.com

Manuscript editor: Klaus Hartfelder as fat body (Petryk et al. 2003). An important action of $20 \mathrm{E}$ in adult female insects is regulation of vitellogenin expression by fat body cells (Raikhel et al. 2002).

Vitellogenin is a yolk protein precursor, but in honey bees also functions as a storage protein supporting synthesis of brood food by the hypopharyngeal glands (Amdam et al. 2003). In adult worker honey bees, vitellogenin titers fall and juvenile hormone ( $\mathrm{JH})$ titers rise at the transition from hive bee to forager (Amdam et al. 2007). The reproductive ground plan hypothesis (RGPH) proposes that regulatory pathways and molecules (such as vitellogenin) used for reproduction in solitary insects are recycled in eusocial insects to regulate division of labor (reviewed in Harwood et al. 2017). Although a role for $\mathrm{JH}$ is an important component of the $\mathrm{RGPH}$, the function of ecdysteroids in adult workers of eusocial species is less clear. 
Despite pro-vitellogenic actions of ecdysteroids in other insects, a delay in the appearance of vitellogenin in hemolymph was produced by treatment of pre-eclosion honey bee queens and workers with ecdysone (Barchuk et al. 2002). The expression of ecdysteroid receptors (EcR) in adult worker brains and ovaries suggests that steroids of ovarian origin can potentially regulate adult behavior and physiology (Nemoto and Hara 2007; Takeuchi et al. 2007; Velarde et al. 2009; Mullen et al. 2014; Fahrbach et al. 2017), but the few studies that have measured hemolymph ecdysteroids in adult workers have reported variable but generally low titers (Robinson et al. 1991; Hartfelder et al. 2002).

One approach to understanding the significance of ecdysteroids in adult worker honey bees has been to focus on variation in size of the source tissue, the ovaries. Worker ovaries typically contain fewer than a dozen ovarioles (Snodgrass 1956; Winston 1987), and individual differences in number of ovarioles, including differences between the right and left ovaries, is commonly observed (Rueppell et al. 2011). The number of ovarioles present at eclosion is established during larval life through an interaction of genetic, nutritional, and hormonal factors (Schmidt Capella and Hartfelder 1998; Rueppell et al. 2011). If the ovaries are the primary source of ecdysteroids in adult workers (Amdam et al. 2010), a simple hypothesis is that workers with larger ovaries will have higher ecdysteroid titers. Comparison of hemolymph ecdysteroids between strains of honey bees selected for high and low pollen hoarding revealed that high-strain honey bees had larger ovaries than low-strain honey bees, but that lowstrain bees had slightly but significantly higher titers of ecdysteroids at adult emergence and on the first day of adult life (Amdam et al. 2010). This difference was not evident on the subsequent 2 days and was reversed on day 4 . These findings are part of a body of work that has provided insight into factors (especially genetic variation, vitellogenin, and $\mathrm{JH}$ ) that regulate adult behavioral development in honey bees (Harwood et al. 2017), but these studies leave unanswered many questions related to function of ecdysteroids in adult workers and the factors that regulate their synthesis, particularly during the later portion of adult life.

The goal of the present study was to ask if extrinsic factors, such as pheromones, contribute to regulation of ecdysteroid titers in adult worker honey bees. We tested the hypothesis that chemical signals produced by actively laying queens modulate ecdysteroid synthesis by worker ovaries. One critical aspect of conditions within queenless $(\mathrm{QL})$ or queenright $(\mathrm{QR})$ colonies was simulated by housing young workers in cages with or without synthetic queen mandibular pheromone (QMP), a blend of five compounds that regulates behavior, physiology, and gene expression in worker honey bees (Hoover et al. 2003; Bortolotti and Costa 2014). Cages were used to isolate QMP as a possible modulatory factor. Contrary to initial predictions, ecdysteroid titers were elevated in young workers from cages in which QMP was present (resembling the QR condition) and low in caged young workers not exposed to QMP (resembling the QL condition). None of these young workers exhibited ovary activation. This result led to further tests of the capacity of QMP to modulate ovarian production of ecdysteroids in honey bee workers. To test for effects on ecdysteroid titers of other cues provided by queens (including mating condition) and the colony environment, we also exposed workers in cages to queens, and studied ecdysteroid titers in workers in QL and QR colonies.

\section{MATERIALS AND METHODS}

\subsection{Honey bees}

Honey bees (Apis mellifera) were collected from apiaries in Forsyth County, NC, USA $\left(36^{\circ}\right.$ $5^{\prime} 59^{\prime \prime} \mathrm{N}$ and $\left.80^{\circ} 14^{\prime} 39^{\prime \prime} \mathrm{W}\right)$. Several different colonies headed by naturally mated queens were used as source colonies. Single-droneinseminated (SDI) queens were obtained as needed from Honey Bee Insemination Service, Washington State University, Pullman, WA, USA. Frames of capped late worker pupae were removed from colonies and held overnight in an incubator $\left(33^{\circ} \mathrm{C}\right)$. Workers less than $12 \mathrm{~h}$ post emergence were collected and rehoused either in cages or field colonies. 


\subsection{Treatments}

Experiment 1: Effects of continuous QMP exposure on ecdysteroid titers of caged worker honey beesNewly emerged worker honey bees $<12 \mathrm{~h}$ post emergence were transferred to Plexiglas ${ }^{\circledR}$ cages $(10 \mathrm{~cm} \times 8 \mathrm{~cm} \times 10 \mathrm{~cm})$. Each cage of 2530 honey bees (same age sisters) was assigned to a treatment group of QMP- (no QMP strip), QMP+ (whole QMP strip), or fractions of a QMP strip. $\mathrm{QMP}+$ cages contained a single 50-mm, fivecomponent synthetic pheromone strip (Phero Tech Inc., Delta, BC, Canada), which mimics exposure to natural QMP, a mixture of 9-oxo-2decenoic acid (9-ODA), the enantiomers of 9hydroxydec-2-enoic acid (9-HDA), methyl phydroxybenzoate (HOB), and homovanillyl alcohol (HVA). Each QMP strip contains 10 queen equivalents of QMP. QMP is not volatile; it is obtained and dispersed by honey bee workers from queens and QMP strips via trophallaxis, antennation, and cuticular contact (Seely 1979; Naumann et al. 1992). Strips were suspended from the back wall of the cage, allowing for ad libitum exposure. QMP- cages contained a single strip of plastic polymer that matched the size and shape of a QMP strip.

Cages were kept in an incubator $\left(28{ }^{\circ} \mathrm{C}, 40 \%\right.$ relative humidity) in darkness. Cages were supplied with ad libitum deionized water, 50\% sucrose $(w / v)$ dissolved in deionized water, and a 1:1 mix of pollen (Brushy Mountain, Moravian Falls, NC, USA) and honey collected from Wake Forest University-maintained hives; pollen was provided to ensure that lack of nutrients did not preclude ovarian activation (Hoover et al. 2006). Daily feeding and cleaning tasks were performed using red light, to which honey bees are insensitive (Peitsch et al. 1992). On day 12 after the cages were established (day 12 of adult life), hemolymph samples were obtained for enzyme immunoassay (EIA) and ovaries were assessed. An additional group of 12-day-old bees was collected at the same time for EIA analysis of the hemolymph content of brains and ovaries, to confirm that the ovaries are the primary tissue source of ecdysteroids in adults, as previously reported (Amdam et al. 2010).
Experiment 2: Age dependence of effects of QMP exposure on ecdysteroid titers of caged worker honey bees Newly emerged worker honey bees were marked on the dorsal thorax with a dot of enamel paint (Testors, Vernon Hills, IL, USA) and transferred to a QR field colony. On days $0,5,12$, and 20, samples of marked workers of known age were collected from the field colony and placed into QMP+ (fulllength 50-mm strip) or QMP- cages with same-age sisters. These ages were selected to cover the period of adult behavioral development through the initiation of foraging (Winston 1987). Five days later, hemolymph samples were obtained for EIA, and ovaries were assessed.

Experiment 3: Comparison of effects on ecdysteroid titers of caged worker honey bees of QMP exposure vs. exposure to naturally mated and SDI queens Newly emerged worker honey bees from a source colony headed by a naturally mated queen were assigned to different treatment cages: $\mathrm{QMP}+, \mathrm{QMP}-, \mathrm{QMP}+$, and $\mathrm{Q}$ (naturally mated queen present); $\mathrm{QMP}-$ and Q, QMP+ and SDI (SDI queen present), and QMP- and SDI. Naturally mated and SDI queens were collected from colonies with eggs in the brood nest. On day 12 , hemolymph samples were obtained for EIA, and ovaries were assessed.

Experiment 4: Ecdysteroid titers of caged SDI workers Newly emerged worker honey bees were obtained from a QR colony established by an SDI queen and assigned to QMP+ or QMP- cages. For comparison of cage and field conditions, an additional 1500 newly emerged honey bees from the same SDI colony were painted and assigned to one of three field colony treatments: the natal SDI colony, an unrelated QR colony (naturally mated queen present), or an unrelated QL colony. The QR and QL colonies were roughly equivalent in terms of population and amount of brood present at the start of the observation period; the QL state was achieved by the removal of the actively laying queen. Workers were collected for sampling from cages and field colonies on days 5,12 , and 20 . 


\subsection{Hemolymph and tissue collections}

After honey bees were immobilized by chilling for $10 \mathrm{~min}$, a size 4 insect pin was used to create an opening in the intersegmental membrane posterior to one of the anterior abdominal sterna; hemolymph was collected at this point by insertion of a 75-mm glass microcapillary tube (Drummond Scientific Company, Broomall, PA, USA). Although sample volumes ranged from 2 to $15 \mu \mathrm{L}$, higher volumes were only obtained occasionally from newly emerged workers and the average volume per worker was approximately $4 \mu \mathrm{L}$. Samples contaminated with fat body or gut contents were discarded, and clear samples were dispensed into siliconized microcentrifuge tubes (Thermo Fisher Scientific, Waltham, MA, USA) containing $100 \mu \mathrm{L}$ of HPLC-grade methanol (Sigma-Aldrich, St. Louis, MO, USA) and stored at $-40{ }^{\circ} \mathrm{C}$ to permit multiple samples to be analyzed in the same assay run. To determine ovarian status, whole ovaries were removed and rinsed with saline, suspended in $2-3 \mathrm{~mL}$ of saline, then assessed using a 5-point scale (Pernal and Currie 2000), in which 0 indicates ovarioles with no signs of swelling, 4 indicates queen-like ovarioles with elongated ooyctes, and intermediate scores are assigned based on degree of swelling and presence of constrictions between follicles, following standard methods (Rueppell et al. 2011). Both ovaries were inspected, and a score was given based on the most advanced development observed. Then, the ovarioles in the right ovary were counted at a magnification of $\times 40$ using a stereomicroscope. In the rare cases, the right ovary was not visible or appeared damaged, and the left ovary was utilized for ovariole counts.

\subsection{Enzyme immunoassay of ecdysteroids}

The EIA has been used to detect ecdysteroids in Drosophila melanogaster (e.g., Ishimoto et al. 2009; Yamanaka et al. 2015) and allows determination of hemolymph titers in individual worker honey bees with commercially available reagents. The polyclonal rabbit IgG antibody on which this EIA is based was raised against 20E (No. 482202, Cayman Chemical, Ann Arbor, MI, USA). The limit of detection of this assay for $20 \mathrm{E}$ is reported as $31 \mathrm{pg} / \mathrm{mL}$ (Bertin Pharma, Montigny-leBretonneux, France). As is the case with other antibodies raised against 20E, this antibody likely binds related steroids such as ecdysone and makisterone A, a characteristic C28 ecdysteroid of honey bees that has been recovered from the ovaries of queen honey bees (Warren et al. 1984; Feldlaufer et al. 1985, 1986). We followed the lead of other authors and conservatively interpreted results as a measure of total ecdysteroids (e.g., Amdam et al. 2010).

Samples stored at $-40{ }^{\circ} \mathrm{C}$ were thawed, vortexed, and held at room temperature for $1 \mathrm{~h}$ prior to running the EIA. Non-hemolymph samples were twice homogenized in $200 \mu \mathrm{L}$ methanol for $30 \mathrm{~s}$ with a handle pestle prior to the 1-h incubation period. Extracted samples were spun at $13,000 \mathrm{~g}$ for $15 \mathrm{~min}$ to remove cellular debris. Supernatants were transferred to siliconized microcentrifuge tubes, then dried using a centrifugal evaporator (Integrated SpeedVac ISS110) at room temperature. Dried samples were resuspended using EIA buffer (No. 400060, Cayman Chemical, Ann Arbor, MI, USA), and the assay was run using a conjugate of $20 \mathrm{E}$ and acetylcholinesterase (No. 482200, Cayman Chemical, Ann Arbor, MI, USA) following the manufacturer's protocol. Signal was detected at $450 \mathrm{~nm}$ after a 2-h incubation using a PerkinElmer EnSpire 2300 Multilabel Reader. Standard curves were prepared using $20 \mathrm{E}$ in quantities from 0.48 to $100 \mathrm{pg} / \mu \mathrm{L}$ (H5142; Sigma-Aldrich, St. Louis, MO, USA), and linear regression was used to calculate the sample ecdysteroid contents based on the standard curve. Results were expressed as total ecdysteroids in picograms per microliter of sample. Analyses were performed in duplicate and averaged. Values falling below the lowest point on the standard curve were reported as zero.

\subsection{Data analysis}

The effects of QMP on ecdysteroid production, ovariole counts, and ovary activation were analyzed with two-way ANOVAs (age and treatments) followed by Tukey-Kramer post hoc tests for pairwise tests. The significance threshold was set at 0.05. Ecdysteroid data were transformed using a standard $\log$ transformation to correct for 
skew (Zar 2010). In Experiment 1, a hierarchical cluster analysis was performed on untransformed ecdysteroid titers using Ward's method to separate individuals exposed to QMP into clusters in an unbiased fashion. Ward's method joins cases into clusters such that the variance within each cluster is minimized (Everitt 1993). Pearson's coefficient $r$ was used to measure the strength of the association between ovariole number and ecdysteroid titer and between ecdysteroids in different tissue samples from the same worker, respectively (IMB SPSS Statistics 22). For ease of interpretation, graphical depictions of data display untransformed values.

\section{RESULTS}

\subsection{QMP exposure was associated with higher ecdysteroid titers in caged adult worker honey bees}

Experiment 1 examined effects of exposure to QMP in caged adult workers. Length of the QMP strip, which is directly related to the surface area available for contact, had a significant effect on ecdysteroid titers (Figure 1). On day 12, mean ecdysteroid titers were significantly elevated in workers exposed to a full QMP strip compared with the QMP- treatment. Titers were significantly elevated in all three intermediate-length QMP conditions relative to the QMP- condition, and titers were significantly elevated in the QMP+ group relative to all three intermediate-length treatments, suggesting that greater access to QMP resulted in higher ecdysteroid titers. There were no differences in activation status of the ovaries nor differences in one-sided (right) ovariole counts (Table I). There was no significant association between ovariole number and ecdysteroid titer (QMP+, $r=-0.01 ; 3 / 4, r=$ $0.55 ; 1 / 2, r=0.59 ; 1 / 4, r=0.247$; QMP-,$r=-$ $0.23 ; p>0.05$ all groups; sample sizes given in Figure 1). Unbiased hierarchical cluster analysis using Ward's method revealed two possible subgroups within all samples exposed to QMP. We refer to the groups identified by cluster analysis as responders and non-responders. In Experiment 1, individual workers exposed to QMP sorted into a group with a mean ecdysteroid titer of $201.8 \pm$



Figure 1. Effect of exposure to QMP on ecdysteroid titers in caged worker honey bees (Experiment 1 ). Ecdysteroid titers $(\mathrm{pg} / \mu \mathrm{L}$; untransformed data; see "Materials and methods" for statistical analysis) sampled after 12 days of exposure to varying intensities of QMP produced by varying the length of the QMP strip in the cage. Overall, the effect of exposing caged workers to QMP was significant: $F_{4}, 70=8.26$, $p<0.01$. Significantly different pairwise comparisons are represented by different letters (Tukey-Kramer post hoc tests, $p<0.05$ ). Box indicates $25-75 \%$; solid line indicates median; dotted line indicates mean; whiskers indicate min-max; open squares indicate outliers. QMP - (no QMP control, $n=17$ ); 1/4 QMP (12.5-mm QMP strip, $n=14), 1 / 2$ QMP (25-mm QMP strip, $n=11), 3 / 4$ QMP (37.5-mm QMP strip, $n=16$ ), and QMP+ (50mm QMP strip, $n=18$ ).

$32.3 \mathrm{pg} / \mu \mathrm{L}$ (putative responders, $n=12$ ) and a group with a mean ecdysteroid titer of $18.2 \pm$ $2.3 \mathrm{pg} / \mu \mathrm{L}$ (putative non-responders, $n=40$ ).

We expected the ovaries to be the most significant post-metamorphic source of ecdysteroids in worker honey bees (Lafont et al. 2005; Amdam et al. 2010). Nervous tissue, however, is a site of steroid production in vertebrates (e.g., Schlinger and Arnold 1991; Schumacher et al. 2015), and a previous report showed that genes encoding two of the cytochrome P450 enzymes used for ecdysteroid synthesis are expressed in the worker honey bee brain (Yamazaki et al. 2011). In a subgroup of subjects from Experiment 1 in which hemolymph, ovaries, and brains were sampled from the same individuals, hemolymph ecdysteroids were significantly $(p<0.01)$ elevated in $\mathrm{QMP}+$ conditions $(115.3 \pm 39.9 \mathrm{pg} / \mu \mathrm{L}, n=$ 27) compared with QMP- conditions (17.7 \pm $5.8 \mathrm{pg} / \mu \mathrm{L}, n=28$ ). Ecdysteroids differed significantly between tissue sources in these workers 
Table I. Ovariole counts and activation scores (Experiments 1 and 2)

\begin{tabular}{llll}
\hline Treatment group & $n$ & Ovarioles $^{\mathrm{a}}$ & Activation $^{\mathrm{b}}$ \\
\hline $\begin{array}{l}\text { Experiment } 1 \\
\text { QMP- }\end{array}$ & 17 & $2.3 \pm 0.2$ & $0.0 \pm 0.0$ \\
1/4 QMP strip & 14 & $3.9 \pm 0.4$ & $0.1 \pm 0.2$ \\
1/2 QMP strip & 11 & $3.0 \pm 0.3$ & $0.02 \pm 0.1$ \\
3/4 QMP strip & 16 & $3.2 \pm 0.3$ & $0.0 \pm 0.0$ \\
QMP+ & 18 & $4.2 \pm 0.4$ & $0.0 \pm 0.0$ \\
Experiment 2 & & & \\
QMP- (0 days) & 31 & $2.4 \pm 0.2$ & $0.0 \pm 0.0$ \\
QMP- (5 days) & 32 & $3.1 \pm 0.3$ & $0.03 \pm 0.03$ \\
QMP- (12 days) & 16 & $2.7 \pm 0.03$ & $0.1 \pm 0.1$ \\
QMP- (20 days) & 18 & $3.4 \pm 0.03$ & $0.1 \pm 0.05$ \\
QMP+ (0 days $)$ & 33 & $2.4 \pm 0.1$ & $0.0 \pm 0.0$ \\
QMP+ (5 days $)$ & 28 & $3.4 \pm 0.2$ & $0.7 \pm 0.5$ \\
QMP+ (12 days $)$ & 12 & $2.5 \pm 0.2$ & $0.6 \pm 0.6$ \\
QMP+ (20 days) & 16 & $4.0 \pm 0.3$ & $0.1 \pm 0.1$ \\
\hline
\end{tabular}

${ }^{a}$ One-sided counts

${ }^{\mathrm{b}}$ Scoring system based on Pernal and Currie (2000)

$(p<0.01)$. In both QMP+ $(102.3 \pm 21.2 \mathrm{pg} / \mu \mathrm{L}$, $n=36)$ and QMP- groups $(69.5 \pm 14.5 \mathrm{pg} / \mu \mathrm{L}$, $n=32$ ), ecdysteroids were higher in ovaries than in brain $(14.4 \pm 3.5, n=39 ; 20.5 \pm 3.8 \mathrm{pg} / \mu \mathrm{L}$, $n=38$ ). Brain ecdysteroids were not correlated with hemolymph titers in either the QMP+ or the QMP- condition $(r=0.33, n=28, p>0.05 ; r=$ $-0.13, n=29, p>0.05)$. Ovarian ecdysteroid levels did not correlate with hemolymph titers in either the QMP+ or QMP- conditions $(r=0.15$, $n=29, p>0.05 ; r=-0.21, n=31, p>0.05)$.

\subsection{Age dependence of QMP-induced ecdysteroid titer elevation}

Experiment 2 investigated the effect of age at exposure to QMP on ecdysteroid titers. There was a significant interaction of age with treatment (Figure 2). QMP+ workers caged immediately after adult emergence had significantly higher hemolymph ecdysteroids $(101.2 \pm 35.4 \mathrm{pg} / \mu \mathrm{L}$, $n=33$ ) compared with their QMP- counterparts $(15.9 \pm 5.0 \mathrm{pg} / \mu \mathrm{L}, n=31, p<0.01)$. No other groups (representing delayed caging) responded

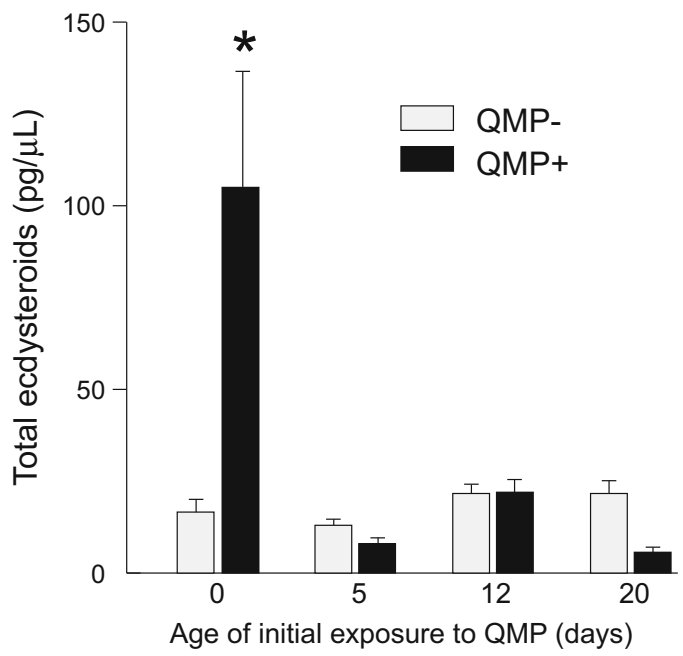

Figure 2. Effect of age of caging on ecdysteroid titers (Experiment 2). There was a significant interaction of age with treatment $\left(F_{3}, 183=5.5, p<0.01\right)$. Figure depicts ecdysteroid titer (mean \pm standard error, $\mathrm{pg} / \mu \mathrm{L}$ ) after 5 days of caging initiated at the ages indicated on the x-axis. Shaded bars indicate housing in cages without exposure to QMP; solid bars indicate housing in cages with a 50-mm QMP strip. Asterisk denotes significant difference in pairwise comparison (Tukey-Kramer post hoc test, $p<0.0001$ ). Sample sizes per age of transfer to cages for QMP- (day $0, n=31$; day $5, n=32$; day 12, $n=16$; day 20, $n=18$ ). Sample sizes per age of transfer to cages for QMP+ (day $0, n=$ 33 ; day $5, n=28$; day $12, n=17$; day $20, n=16$ ).

to 5 days of QMP exposure in cages with elevated hemolymph ecdysteroids. Neither age $\left(F_{3,183}=\right.$ $1.4, p>0.05)$ nor QMP treatment $\left(F_{1,183}=0.25\right.$, $p>0.05)$ independently impacted ecdysteroid titers. There were no differences in ovary activation or one-sided ovariole counts in any of the workers sampled (Table I).

\subsection{Interaction of QMP with other signals provided by queens}

Experiment 3 examined effects of presence of a queen and queen mating condition on worker responses to QMP. The goal was to test the hypothesis that other signals (chemical, behavioral) produced by queens can modify (enhance or inhibit) the effects of QMP on ecdysteroid titers. There was a significant effect of queen type (naturally mated 
vs. SDI) on ecdysteroid titers (Figure 3). Bees caged with an SDI queen had significantly lower ecdysteroids in the QMP+ and QMP- conditions compared with workers housed with naturally mated queens. Workers caged with naturally mated queens did not differ significantly from cages without queens. There were no differences in ovary activation or ovariole count in any of the groups of workers sampled (data available in electronic supplementary material).

\subsection{Social modulation of QMP effects on ecdysteroid titers}

Experiment 4 compared ecdysteroid production in the presence of an SDI queen in workers reared in two different cage environments (QMP+ and QMP-) and three different colony environments (natal SDI colony, a full-size QR colony, and a full-size QL colony). The goal was to examine responses of workers who are full sisters under different cage and colony conditions. There was a

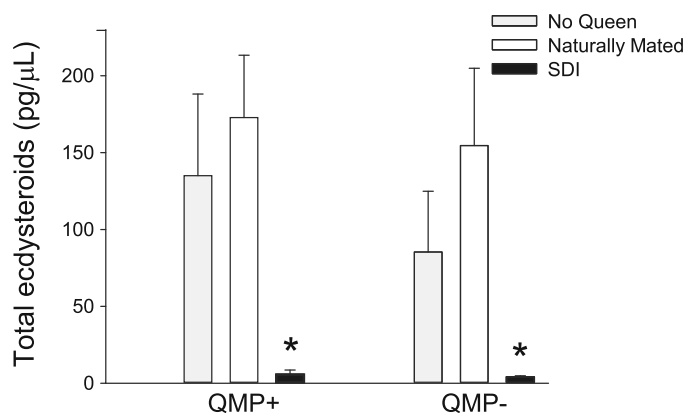

Figure 3. Effect of queen condition on ecdysteroid titers in workers caged with or without QMP (Experiment 3 ). There was a significant effect of queen type $\left(F_{2}, 78=8.57, p<0.01\right)$ on ecdysteroid titers. Figure depicts ecdysteroid titers (mean \pm standard error, $\mathrm{pg} / \mu \mathrm{L}$ ) measured in samples collected 12 days after newly emerged workers were caged. Shaded bars indicate housing in a cage with no queen present; open bars indicate housing with a naturally mated queen; solid bars indicate housing with an SDI queen. Asterisk denotes significant difference in pairwise comparisons within the QMP+ and QMP- conditions (TukeyKramer post hoc test, $p<0.01)$. Sample sizes for QMP+ conditions: no queen, $n=10$; naturally mated queen, $n=14$; SDI queen, $n=12$. Sample sizes for QMP- conditions: no queen, $n=10$; naturally mated queen, $n=19$; SDI queen, $n=14$. significant effect of treatment age on ecdysteroid titers in QMP+ vs. QMP- cages and QR vs. QL colonies (Figure 4). Additionally, there was a significant effect of interaction between treatment and age $\left(F_{6,281}=20.0, p<0.01\right)$. In contrast to results

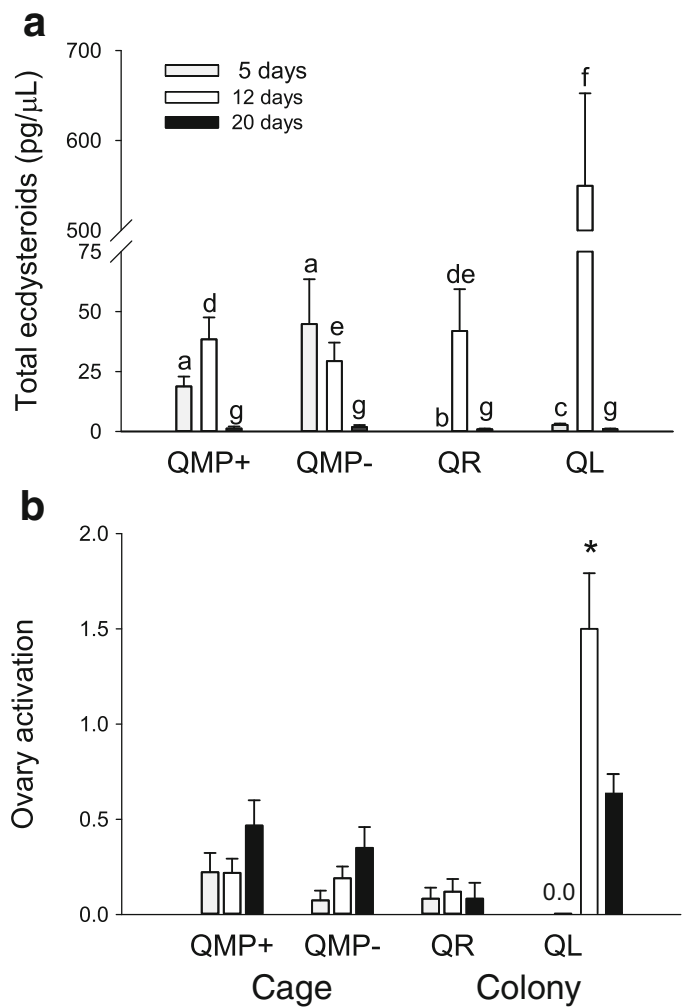

Figure 4. Effect of age and treatment on ecdysteroid production and ovary activation compared for field and laboratory conditions (Experiment 4). All workers in this experiment were offspring of a single SDI queen. a Ecdysteroid titers (mean \pm standard error, $\mathrm{pg} / \mu \mathrm{L}$ ) in daughters of an SDI queen reared in cages with or without QMP and in either a host QR or QL field colony. Zero $(0)$ readings were below threshold of the assay. Different letters denote significant differences, comparisons across treatments by day, $p \leq 0.05$. b Ovary activation by treatment (mean \pm standard error). Asterisk denotes significant difference in Tukey-Kramer post hoc test within the QL condition, $p<0.05$. Sample sizes for QMP+ conditions: 5 days $(n=18) ; 12$ days $(n=32)$; 20 days $(n=15)$. Sample sizes for QMPconditions: 5 days $(n=27) ; 12$ days $(n=44) ; 20$ days $(n=20)$. Sample sizes for QR conditions: 5 days $(n=$ $24), 12$ days $(n=25) 20$ days $(n=12)$. Sample sizes for QL conditions: 5 days $(n=17)$; 12 days $(n=14)$; 20 days $(n=33)$. 
obtained in Experiment 2, which used progeny of a naturally mated queen, no difference was found between hemolymph ecdysteroids of caged QMP+ and QMP- workers sampled on day 5. Titers, however, were significantly elevated on day 5 relative to those in SDI workers reared in field colonies under both QR and QL conditions.

Ecdysteroid titers were significantly higher in SDI workers reared in QMP+ cages on day 12 compared with titers of workers reared in QMPcages. SDI workers housed in the QR colony did not differ significantly from either cage treatment when sampled on day 12. By contrast, ecdysteroids were significantly elevated on day 12 in the QL colony relative to all other day 12day-old workers sampled in these studies. There was a significant effect of treatment $\left(F_{3,279}=\right.$ $25.9, p<0.01)$ with age $\left(F_{2}, 279=25.3\right.$, $p<0.01)$ on ovarian activation. The 12-day-old SDI worker QL group contained the only workers sampled in these studies with significant morphological signs of ovary activation on the Pernal and Currie scale $(1.5 \pm 0.29 ; p<0.01)$.

Workers raised in their natal SDI colony displayed a trend not observed in any other group of workers continuously exposed to QMP since emergence: age had a significant effect on ecdysteroid titers in the QR SDI colony, but the effect was evident only on day 5 (data available in electronic supplementary material). In these workers, ecdysteroid levels decreased from day $5(56.2 \pm 7.9 \mathrm{pg} / \mu \mathrm{L}, n=19)$ to day $12(15.4 \pm$ $6.40 \mathrm{pg} / \mu \mathrm{L}, n=15 ; p<0.01)$.

\section{DISCUSSION}

\subsection{QMP exposure modulated ecdysteroid production in young adult worker honey bees}

Current views of a limited role for ecdysteroids in adult worker physiology reflect the fact that an individual honey bee can provide only a tiny volume of hemolymph for analysis and can provide that sample only once. The result is a sparse literature based on one-time samples, raising the possibility that subtle but biologically relevant individual differences and changes over time important for endocrine signaling may be missed. Fluctuating conditions in field colonies are another source of variation in hormone measures across studies. Cages allow for stable nutrition, controlled proximity to a stable QMP source, and control of social influences known and unknown (Figure 5). By pairing an EIA that permits analysis of samples from individual workers with rearing in cages and age-matched comparisons, we found that continuous exposure to QMP from the

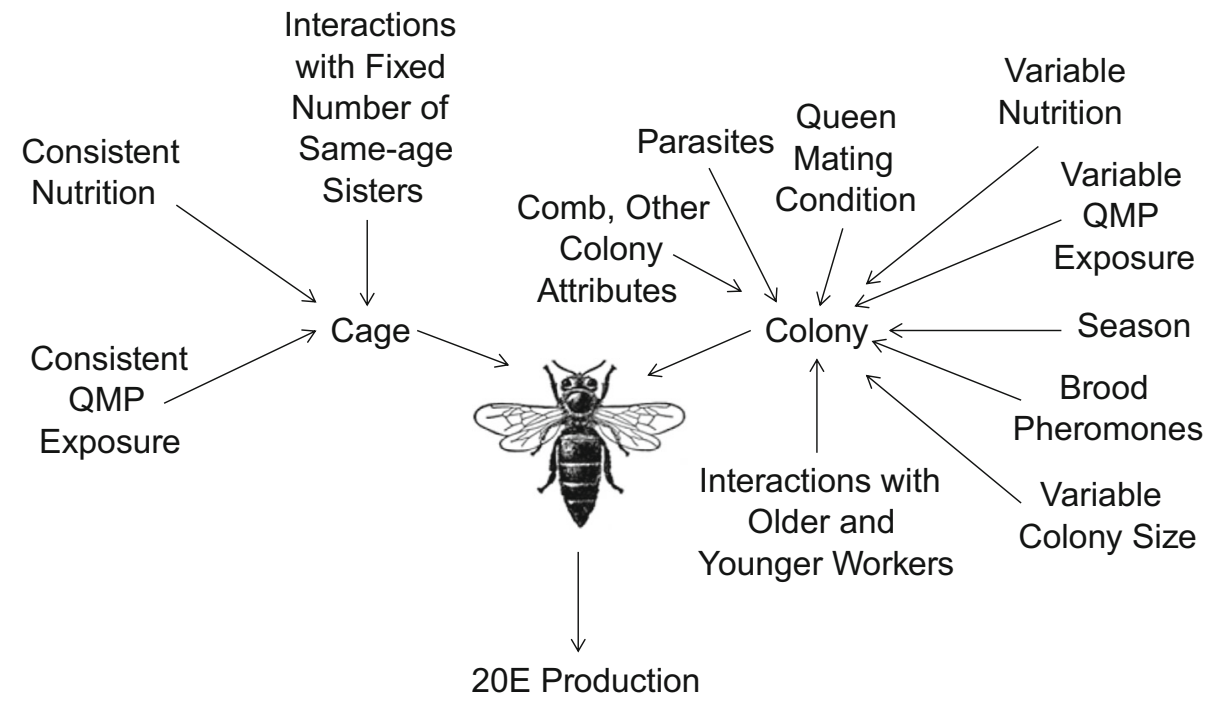

Figure 5. A summary of possible environmental (including social) influences on ecdysteroid production in adult worker honey bees studied under cage and colony conditions. 
beginning of adult life elevated ecdysteroids in adult worker honey bees. This result is significant because treatment of workers with $20 \mathrm{E}$ has been shown to alter vitellogenin titers (Barchuk et al. 2002), and vitellogenin in turn regulates many aspects of worker behavior and physiology (Harwood et al. 2017).

In QMP+ groups, a subset of workers exhibited ecdysteroid titers as much as 10 times higher than the lowest observed values within the same treatment group, and post hoc statistical analysis indicated the possibility of two clusters within QMP+ groups. We suggest that responders (defined as individuals that show elevated ecdysteroid titers in the presence of QMP) and non-responders (individuals that do not show elevated titers in the presence of QMP) possibly represent different genotypes but may also reflect differences in epigenetic factors. The pheromone response threshold has been previously shown to be influenced by individual differences such as ovary size, social behavior, and age of exposure (Amdam et al. 2009; Pankiw and Page Jr 1999; Traynor et al. 2014; Vergoz et al. 2009). A study in which responses to QMP presentation were observed in caged workers on the first 8 days of adult life revealed colony-level and individual differences in performance of the QMP-evoked retinue response, as well as differences in QMP-evoked brain gene expression profile (Kocher et al. 2010). It will be interesting to explore the relationship between individual differences in worker behavioral and brain gene expression responses to QMP and ecdysteroid titers, as circulating ecdysteroids are a major link between the ovaries and the brain.

\subsection{Ecdysteroid titers were unrelated to ovary activation and ovariole number in caged workers}

The assumed role of ecdysteroids in the reproductive physiology of honey bees is based primarily on studies of non-hymenopterans (Schwartz et al. 1989; Kozlova and Thummel 2000). Elevated ecdysteroids were previously reported for both honey bee queens and workers sampled from colonies containing laying workers, but the ovaries of the laying workers were not examined in that study (Robinson et al. 1991). Here we show that workers with elevated ecdysteroid titers on day 12 did not exhibit morphological signs of ovary activation. This is in accord with results reported by Hartfelder et al. (2002), who reported no correlation between ecdysteroid titers in worker honey bees and activation of the ovaries.

Ovariole number is determined in larval honey bees by multiple genotypic, nutritional, and hormonal factors (Evans and Wheeler 1999; Schmidt Capella and Hartfelder 2002; Rueppell et al. 2011; and many others). There is a reported positive association between worker ovariole number and tendency to show signs of morphological activation of the ovaries (Page et al. 2006; Amdam et al. 2006; Tsuruda et al. 2008; Traynor et al. 2014). We did not find a correlation between ovariole number and ecdysteroid titer, and as noted, morphological signs of activation were not observed in workers with elevated ecdysteroid titers apart from a group of 12-day-old workers housed in a QL colony. This suggests separation of ecdysteroid production and reproductive capacity in non-laying workers. A recent study showing that worker ovariole number can decrease with age does not bias our findings because our comparisons were based on age-matched groups (Ronai et al. 2017).

The omission of QMP from cages mimics a key aspect of the QL state, so it is notable that we did not see activation of the ovaries in QMPcaged groups. This disparity between our results and others that have reported ovarian activation in workers (e.g., Hoover et al. 2003; Tsuruda et al. 2008; Traynor et al. 2014) may reflect slight variations in rearing conditions but also the relatively small size of worker ovaries in the unselected colonies we used (see also Ronai et al. 2017). Mean ovariole counts in these studies ranged from $1.5 \pm 0.3$ to $4 \pm 0.3$ ovarioles per side, whereas studies reporting significant numbers of activated worker ovaries have reported ovariole counts as high as $6 \pm 3.6$ and $>8$ (Tsuruda et al. 2008; Traynor et al. 2014). Our smaller number of ovarioles and reduced range of variation are instead consistent with the study of Oldroyd and Beekman (2008), who reported lower levels of 
ovarian activation and smaller ovary size overall in workers from naturally mated queens collected from field colonies. Because we work with commercially available lines that are not heavily selected for specific traits, we suggest our results reflect a more typical condition for ovaries in worker honey bees. Genetic sources of variation, such as directional behavioral selection for pollen hoarding (Amdam et al. 2010) or patriline selection for traits such as anarchy (Thompson et al. 2008), have been shown to increase the tendency of workers to activate their ovaries (Backx et al. 2012). Heavy selection for behavioral traits may result in loss or masking of subtle aspects of physiological regulation. Also, many studies that describe ovarian activation typically report low scores, rarely exceeding a 1 (slight swelling of the ovarioles) or 2 (moderate swelling of the ovarioles) on the Pernal and Currie (2000) scale. Other investigators have taken a more conservative approach in classifying only ovarioles with developed ooyctes and clear signs of yolk deposition as activated (Malka et al. 2007). Ovarian activation measured as swelling alone appears not to be a useful predictor of hemolymph titers of ecdysteroids.

Ovarian ecdysteroid content of caged 12day-old workers was not correlated with hemolymph ecdysteroid titer. In a prior study of young (1-4-day-old) workers reared in a field colony, a positive correlation was reported between a combined, longer-term measure of ovarian ecdysteroid production (medium from ovaries cultured over a period of $18 \mathrm{~h}$ pooled with homogenate of the cultured ovaries), suggesting that a cumulative assay is required to detect a relationship (Amdam et al. 2010); the same study reported that negligible ecdysteroids were recovered from brainconditioned medium pooled with brain homogenates. Taking these findings together with the present results, we support the view that it is unlikely that the honey bee brain is a source of circulating ecdysteroids. We suggest that the report by Yamazaki et al. (2011) of brain ecdysteroid contents and brain expression of cytochrome P450 enzymes critical for steroid biosynthesis indicates a paracrine rather than an endocrine role for neural ecdysteroids.

\subsection{Age and social context modulated ecdysteroid titers in the presence and absence of QMP}

Caged workers needed to have proximity to a QMP source from the first day of adult life to show elevated ecdysteroid titers; delayed introduction to the same environment did not produce the same response, as older caged workers appeared refractory to QMP. Other responses of workers to QMP also display age dependency. For example, attractiveness of QMP wanes as workers age: young workers are attracted to QMP while older workers are repelled (Vergoz et al. 2009). Using microarrays and mRNA quantification by qRT-PCR, Grozinger (2003) showed that exposure to QMP differentially regulated expression of several hundred genes in young nurse bees vs. older foragers. The basis for the age dependency of these responses is currently unknown.

Social context also modulated responses to QMP. Ecdysteroid titers on day 12 in QR colonies were similar to those observed in $\mathrm{QMP}+$ cages, but lower than those in the QL colony. A QL colony produced the highest levels of ovary activation, with a subset of 12-day-olds showing developed oocytes. Ecdysteroid titers were also elevated in this group, revealing a role for cues from the colony distinct from cues provided by a queen in regulation of this hormone.

Surprisingly, ecdysteroid titers were low in workers in cages with an SDI queen regardless of exposure to additional QMP, while naturally mated queens induced the same trend as seen with synthetic QMP exposure. Additionally, a unique ecdysteroid titer profile and a trend towards increased ovarian activation over time were observed in field colonies headed by SDI queens. This is similar to findings showing higher levels of ovarian activation in SDIheaded colonies (Mattila et al. 2012). The relationship of SDI status to ovarian activation, however, appears to be variable, as other studies have shown that workers are less likely to develop their ovaries in colonies headed by SDI queens (Peso et al. 2013; Hoover et al. 2003). 
Honey bee queens produce multiple pheromones as well as other compounds not currently considered pheromones (Slessor et al. 1988; Hoover et al. 2003; Peso et al. 2013). SDI queens and naturally mated queens have the potential to differ along many dimensions beyond the contents of their spermathecas, including differences in the early handling of SDI queens related to insemination (Cobey 2007). Given that caged SDI queens had a different effect on worker ecdysteroid titers than did caged naturally mated queens, we suggest that chemical signals differentially produced by SDI queens may be responsible for the effects revealed in the controlled cage environment.

\subsection{Summary}

Our data show that QMP exposure modulates ecdysteroid titers in worker honey bees. A subset of workers (responders) reared outside of the hive environment responds to consistent and intense QMP exposure with elevated ecdysteroid titers. These circulating ecdysteroids have the capacity to impact behavior and physiology through regulation of brain gene expression via brain ecdysteroid receptors (Velarde et al. 2009; Fahrbach et al. 2017), independent of reproductive function. This view is consistent with our finding that the traditional morphological signs of the early stages of ovarian activation cannot be used as predictors of ecdysteroid titers in worker honey bees.

\section{ACKNOWLEDGEMENTS}

This research was supported by a grant from the National Science Foundation (contract grant number IOS1256878) and a Wake Forest University Center for Molecular Signaling grant to AMT. E. Snow and S. Hathaway assisted with beekeeping; O. Rueppell provided training in ovary dissection; S. Barbato, J. Lee, A. Kennedy, and K. Stevenson assisted with sampling.

\section{AUTHORS' CONTRIBUTION}

Both authors have contributed equally to the work. Both authors read and approved the final manuscript.

\section{COMPLIANCE WITH ETHICAL STANDARDS}

Conflict of interest The authors declare that they have no conflict of interest.

\section{OPEN ACCESS}

This article is distributed under the terms of the Creative Commons Attribution 4.0 International License (http://creativecommons.org/licenses/by/4.0/), which permits unrestricted use, distribution, and reproduction in any medium, provided you give appropriate credit to the original author(s) and the source, provide a link to the Creative Commons license, and indicate if changes were made.

La phéromone mandibulaire de la Reine module les titres des ecdystéroïdes de l'hémolymphe chez les ouvrières adultes d'Apis mellifera

Ecdystéroïdes /ovaire / phéromone mandibulaire de la reine / orphelin / présence de reine

Das Mandibelpheromon der Königin moduliert den Ecdysteroidtiter der Hämolymphe von adulten Apis mellifera Arbeiterinnen

Ecdysteroide / ovar / Mandibelpheromon de Königin / weisellos / weiselrichtig

\section{REFERENCES}

Amdam, G.V., Norberg, K., Hagen, A., Omholt, S.W. (2003) Social exploitation of vitellogenin. Proc. Natl. Acad. Sci. USA 100 (4) 1799-1802

Amdam, G.V., Csondes, A., Fondrk, M.K., Page, R.E., Jr. (2006) Complex social behaviour derived from maternal reproductive traits. Nature 439, 76-78

Amdam, G.V., Nilsen, K.A., Norberg, K., Fondrk, M.K., Hartfelder, K. (2007) Variation in endocrine signaling underlies variation in social life history. Am. Nat. 170 (1) $37-46$

Amdam, G.V., Rueppell, O., Fondrk, M.K., Page, R.E. Jr, Nelson, C.M. (2009) The nurse's load: early-life exposure to brood-rearing affects behavior and lifespan in honey bees (Apis mellifera). Exp. Gerontol. 44 (6-7), 467-471

Amdam, G. V., Page, R. E., Fondrk, K. M., Brent C. S. (2010) Hormone response to bidirectional selection on social behavior. Evol. Dev. 12 (5), 428-436 
Backx, A. G., Guzmán-Novoa, E., Thompson, G.J. (2012) Factors affecting ovary activation in honey bee workers: a meta-analysis. Insectes Soc. 59 (3), 381-388

Barchuk, A.R., Bitondi, M.M., Simões, Z.L. (2002) Effects of juvenile hormone and ecdysone on the timing of vitellogenin appearance in hemolymph of queen and worker pupae of Apis mellifera. J. Insect Sci. 2 (1). Available online: insectscience.org/2.1

Bortolotti, L., Costa, C. (2014) Chemical communication in the honey bee society, in: Mucignat-Caretta, C. (Ed.), Neurobiology of chemical communication. CRC Press, Boca Raton, pp. 147-210

Brown, M.R., Sieglaff, D.H., Rees, H.H. (2009) Gonadal ecdysteroidogenesis in Arthropoda: Occurrence and regulation. Annu. Rev. Entomol. 54, 105-125

Cobey, S.W. (2007) Comparison studies of instrumentally inseminated and naturally mated honey bee queens and factors affecting their performance. Apidologie 38 (4), 390-410

Dai, J. D., Gilbert, L.I. (1997) Programmed cell death of the prothoracic glands of Manduca sexta during pupaladult metamorphosis. Insect Biochem. Molec. Biol. 27 (1), 69-78

Evans, J. D., Wheeler, D.E. (1999) Differential gene expression between developing queens and workers in the honey bee, Apis mellifera. Proc. Natl. Acad. Sci. USA 96 (10), 5575-5580

Everitt, B. (1993). Cluster analysis (3rd edition). Arnold, London

Fahrbach, S.E., Trawinski, A.M., Velarde, R.A. (2017) Nervous system actions of insect development hormones in adult insects, in: Pfaff, D.W., Joels, M., Hormones, Brain and Behavior, 3rd edn. Academic Press Ltd., London, pp. 349-366

Feldlaufer, M.F., Herbert, E.W. Jr., Svoboda, J.A., Thompson, M.J., Lusby, W.R. (1985) Makisterone A: The major ecdysteroid from the pupa of the honey bee, Apis mellifera. Insect Biochem. 15 (5): 597-600

Feldlaufer, M.F., Svoboda, J.A., Herbert, E. W., Jr. (1986) Makisterone A and 24-methylenecholesterol from the ovaries of the honey bee, Apis mellifera L. Experientia 42, 200-201

Grozinger, C. M. (2003) Pheromone-mediated gene expression in the honey bee brain. Proc. Natl. Acad. Sci. USA 100, 14519-14525

Hartfelder, K., Bitondi M.M., Santana, W.C. Simões, Z.L. (2002) Ecdysteroid titer and reproduction in queens and workers of the honey bee and of a stingless bee: Loss of ecdysteroid function at increasing levels of sociality? Insect Biochem. Molec. Biol. 32 (2), 211-216

Harwood, G.P., Ihle, K.E., Salmela, H., Amdam, G.V. (2017) Regulation of honeybee worker (Apis mellifera ) life histories by vitellogenin, in: Pfaff, D.W., Joels, M., Hormones, Brain and Behavior, $3^{\text {rd }}$ edition. Academic Press Ltd., London, pp. 403-420

Hoover, S.E.R., Keeling, C.I., Winston, M.L., Slessor, K. N. (2003) The effect of queen pheromones on worker honey bee ovary development. Naturwissenschaften 90 (10), 477-480
Hoover, S.E., Higo, H.A., Winston, M.L. (2006) Worker honey bee ovary development: Seasonal variation and the influence of larval an adult nutrition. J. Comp. Physiol. B 176 (1), 55-63

Ishimoto, H., Sakai, T., Kitamoto, T. (2009). Ecdysone signaling regulates the formation of long-term courtship memory in adult Drosophila melanogaster. Proc. Natl. Acad. Sci. USA 106 (15), 6381-6386

Kocher, S.D., Ayroles, J.F., Stone, E.A., Grozinger, C.M. (2010) Individual variation in pheromone response correlates with reproductive traits and brain gene expression in worker honey bees. PLoS ONE 5 (2), e9116

Kozlova, T., Thummel, C.S. Steroid regulation of postembryonic development and reproduction in Drosophila. (2000) Trends Endocrinol. Metabol. 11 (7), 276-280

Lafont, R., Dauphin-Villemant, C., Warren, J., Rees, H.H. (2005) Ecdysteroid chemistry and biochemistry, in: Gilbert, L.I., Iatrou, K., Gill, S. (Eds.). Comprehensive molecular insect science, vol. 3, Elsevier, New York, pp 125-196

Malka, O., Shnieor, S. Hefetz, A., Katzav-Gozansky, T. (2007). Reversible royalty in worker honeybees (Apis mellifera) under the queen influence. Behav. Ecol. Sociobiol. 61 (3), 465-473

Mattila, H.R., Kern, R., Smith, M.L. (2012) Promiscuous honey bee queens increase colony productivity by suppressing worker selfishness. Current Biol. 22 (21), 2027-2031

Mullen, K.M., Daley, M., Backx, A.G., Thompson, G.J. (2014) Gene co-citation networks associated with worker sterility in honey bees. BMC Systems Biol. $\mathbf{8}, 38$

Naumann, K., Winston, M.L., Slessor, K.N., Prestwich, G.D., Latli, B. (1992) Intra-nest transmission of aromatic honey bee queen mandibular gland pheromone components: Movement as a unit. The Canadian Entomol. 124 (5), 917-934

Nemoto, M., Hara, K. (2007) Ecdysone receptor expression in developing and adult mushroom bodies of the ant Camponotus japonicus. Dev. Genes Evol. 217 (9): 619-627

Niwa, R., Niwa, Y.S. (2014) Enzymes for ecdysteroid biosynthesis: Their biological functions in insects and beyond. Biosci. Biotechnol. Biochem. 78 (8), 1283-1292

Oldroyd, B. P., Beekman, M. (2008). Effects of selection for honey bee worker reproduction on foraging traits. PloS Biology. 6 (3), e56

Page, R.E., Scheiner, R., Erber, J., Amdam, G.V. (2006) The development and evolution of division of labor and foraging specialization in a social insect (Apis mellifera L.) Curr. Topics Dev. Biol. 74, 253-286

Pankiw, T., Page Jr, R.E. (1999) The effect of genotype, age, sex, and caste on response thresholds to sucrose and foraging behavior of honey bees (Apis mellifera L.). J. Comp. Physiol. A 185 (2), 207-213

Peitsch, D., Fietz, A., Hertel, H., Souza, J., Ventura, D.F., Menzel, R. (1992) The spectral input systems of 
hymenopteran insects and their receptor-based colour vision. J. Comp. Physiol. A, 170 (1), 23-40

Pernal, S., Currie, R. (2000) Pollen quality of fresh and 1 year old single pollen diets for worker honey bees (Apis mellifera L.). J. Insect Physiol. 56, 132-137

Peso, M., Nino, E.L., Grozinger, C.M., Barron, A.B. (2013) Effect of honey bee queen mating condition on worker ovary activation. Insectes Soc., 60 (2), 123-133

Petryk, A., Warren, J.T., Marques, G., Jarcho, M.P., Gilbert, L.I., Kahler, J., Parvy, J.P., Li, Y., Dauphin-Villemant, C., O'Connor, M.B. (2003) Shade is the Drosophila P450 enzyme that mediates the hydroxylation of ecdysone to the steroid insect molting hormone 20hydroxyecdysone. Proc. Natl. Acad. Sci. USA 100 (24), 13773-13778

Raikhel, A. S., Kokoza, V.A., Zhu, J., Martin, D., Wang, S., Li, C., Sun, G., Ahmed, A., Dittmer, N., Attardo, G. (2002) Molecular biology of mosquito vitellogenesis: From basic studies to genetic engineering of antipathogen immunity. Insect Biochem. Molec. Biol. 32 (10), 1275-1286

Robinson, G., Strambi, C., Strambi, A., Feldlaufer, M. (1991) Comparison of juvenile hormone and ecdysteroid haemolymph titres in adult worker and queen honey bees (Apis mellifera). J. Insect Physiol. 37 (12), 929-935

Ronai, I., Allsopp, M.H., Tan, K., Dong, S., Liu, X., Vergoz, V., Oldroyd, BP (2017) The dynamic association between ovariole loss and sterility in adult honeybee workers. Proc. Roy. Soc. B 284 : 20162693

Rueppell, O., Metheny, J.D., Linksvayer, T., Fondrk, M.K., Page, R.E. Jr., Amdam, G.V. (2011) Genetic architecture of ovary size and asymmetry in European honeybee workers. Heredity 106 (5): 894-903

Schlinger, B.A., Arnold, A.P. (1991) Brain is the major site of estrogen synthesis in a male songbird. Proc. Natl. Acad. Sci. USA 88 (10): 4191-4194

Schmidt Capella, I.C., Hartfelder, K. (1998) Juvenile hormone effect on DNA synthesis and apoptosis in castespecific differentiation of the larval honey bee (Apis mellifera L.) ovary. J. Insect Physiol. 44 (5-6): 385391

Schmidt Capella, I.C., Hartfelder, K. (2002) Juvenilehormone-dependent interaction of actin and spectrin is crucial for polymorphic differentiation of the larval honey bee ovary. Cell Tissue Res. 307 (2): 265-272

Schumacher, M., Guennoun, R., Mattern, C., Oudinet, J.P., Labombarda, F., De Nicola, A.F., Liere, P. (2015) Analytical challenges for measuring steroid responses to stress, neurodegeneration and injury in the central nervous system. Steroids 103, 42-57

Schwartz, M.B., Kelly, T.J., Woods, C.W., Imberski, R.B. (1989) Ecdysteroid fluctuations in adult Drosophila melanogaster caused by elimination of pupal reserves and synthesis by early vitellogenic ovarian follicles. Insect Biochem. 19 (3), 243-249

Seely, T.D. (1979) Queen substance dispersal by messenger workers in honeybee colonies. Behav. Ecol. Sociobiol. 5 (4), 391-415

Slessor, K. N., Kaminski, L., King, G.G.S., Borden, J.H., Winston, M.L. (1988). Semiochemical basis of the retinue response to queen honey bees. Nature $\mathbf{3 3 2}$, $54-56$

Snodgrass, R.E. (1956). Anatomy of the honey bee. Comstock Publishing Associates, New York

Takeuchi, H., Paul, R.K., Matsuzaka, E., Kubo, T. (2007) $E c R$ - $A$ expression in the brain and ovary of the honeybee (Apis mellifera L.). Zool. Sci. 24 (6), 596-603

Thompson, G. J., Kucharski, R., Maleszka, R., and Oldroyd, B.P. (2008) Genome-wide analysis of genes related to ovary activation in worker honey bees. Insect Molec. Biol. 17 (6), 657-665

Traynor, K. S., Le Conte, Y., Page, R. E. (2014) Queen and young larval pheromones impact nursing and reproductive physiology of honey bee (Apis mellifera) workers. Behav. Ecol. Sociobiol. 68 (12), 2059-2073

Tsuruda, J. M., Amdam, G. V., Page, R. E. (2008) Sensory response system of social behavior tied to female reproductive traits. PLoS One 3(10):e3397. https://doi. org/10.1371/journal.pone.0003397

Velarde, R.A., Robinson, G.E., Fahrbach, S.E. (2009) Coordinated responses to developmental hormones in the Kenyon cells of the adult worker honey bee brain (Apis mellifera L.). J. Insect Physiol. 55 (1): 59-69

Vergoz, V., McQuillan, H.J., Geddes, L.H., Pullar, K., Nicholson, B.J., Paulin, M.G., Mercer, A.R. (2009) Peripheral modulation of worker bee responses to queen mandibular pheromone. Proc. Natl. Acad. Sci. USA 106 (49), 20930-20935

Warren, J.T., Smith, W., Gilbert, L.I. (1984) Simplification of the ecdysteroid radioimmunoassay by the use of Protein A from Staphylococcus aureus. Experientia 40 (4): 393-394

Winston, M.L. (1987) The biology of the honey bee. Harvard University Press, Cambridge

Yamanaka, N., Marqués, G., O’Connor, M.B. (2015) Vesicle-mediated steroid hormone secretion in Drosophila melanogaster. Cell 163, 907-919

Yamazaki, Y., Kiuchi, M., Takeuchi, H., Kubo, T. (2011). Ecdysteroid biosynthesis in workers of the European honeybee Apis mellifera L. Insect Biochem. Molec. Biol. 41 (5), 283-293

Zar, J.H. (2010) Biostatistical analysis, 5th edn, Pearson, New York 